\title{
USO DE RESÍDUOS EM PAVIMENTOS DE ESTRADAS FLORESTAIS: CARACTERIZAÇÃO DE SOLOS E MISTURAS SOLO-GRITS ${ }^{1}$
}

\author{
Reginaldo Sérgio Pereira ${ }^{2}$, Carlos Cardoso Machado ${ }^{3}$ e Carlos Alexandre Braz de Carvalho ${ }^{4}$
}

\begin{abstract}
RESUMO - Este artigo aborda uma etapa preliminar de um estudo direcionado às propriedades de engenharia de misturas de solos e aditivos químicos, para fins de aplicação em estradas florestais. Trabalhou-se com amostras de dois solos da Zona da Mata Norte de Minas Gerais, Brasil, de texturas arenosa e argilosa, estabilizadas com o resíduo industrial denominado "grits" proveniente de indústria de celulose. Foram realizados ensaios geotécnicos nos solos e nas misturas solo-aditivo químico, como se segue: análise granulométrica, peso específico dos sólidos, limites de Atterberg e compactação. Os resultados encontrados dos ensaios de laboratório revelaram que o resíduo apresentou bom desempenho como agente estabilizante, alterando significativamente a plasticidade e os parâmetros de compactação dos solos analisados.
\end{abstract}

Palavras-chave: Estabilização de solos, resíduo industrial, caracterização geotécnica.

\section{APPLICATION OF WASTE IN FOREST ROAD PAVEMENTS: CHARACTERIZATION OF SOILS AND SOIL-GRITS MIXTURES}

\begin{abstract}
This paper addresses data from a research program in development on engineering properties of soil-chemical stabilizer mixtures for forest road application. Soil samples from sandy and clayey soils from the Zona da Mata Norte of Minas Gerais, Brazil, stabilized with grits (waste from cellulose industries) were used throughout the study. The laboratory-testing program comprised the following geotechnical tests: sieve analysis, density of solid particles, Atterberg limits and compaction. Data supported the good behavior of waste as stabilizer agent changing significantly soil plasticity and compaction parameters.
\end{abstract}

Keywords: Soil stabilization, industrial waste and geotechnical characterization.

\section{INTRODUÇÃO}

Na engenharia rodoviária, tem-se conhecimento de diversos materiais com comprovada eficiência para serem usados em camadas constitutivas de pavimentos. O emprego de produtos naturais, como cascalho e brita, e de misturas de solos e estabilizantes químicos tradicionais, como cal, cimento e betume (asfaltos e alcatrões), é freqüentemente associado à existência de jazidas de empréstimo em áreas próximas ao empreendimento e às questões de caráter técnicoeconômico-ambiental.

Nogami et al. (1980), em menção ao emprego da estabilização solo-cal no Brasil, destacaram que a engenharia rodoviária brasileira deveria tender à busca de um refinamento técnico que contemplasse a utilização dos solos segundo técnicas as mais diversas e

\footnotetext{
${ }^{1}$ Recebido em 22.11.2005 e aceito para publicação em 05.04.2006.

${ }^{2}$ Departamento de Engenharia Florestal da Universidade de Brasília, Brasília-DF. E-mail: <reginaldosp1 @ yahoo.com.br>.

${ }^{3}$ Departamento de Engenharia Florestal da UFV, Viçosa-MG, Brasil. E-mail: <machado@ufv.br>.

${ }^{4}$ Departamento de Engenharia Civil da UFV, Viçosa-MG, Brasil. E-mail: <cabraz@ufv.br>.
} 
sofisticadas. Ampliar o leque de soluções tradicionais disponíveis tem sido o grande desafio para técnicos e pesquisadores que atuam na pavimentação de rodovias.

No Brasil, correntemente, o aproveitamento de resíduos ou subprodutos industriais aparece como uma alternativa técnica promissora de baixo custo. Entretanto, conforme relatado por Fernandes (2000), isso requer o desenvolvimento de programas extensivos de experimentação de laboratório e campo, de forma a alcançar os padrões de engenharia exigidos pelas normas técnicas. Em se tratando de programas de laboratório, a primeira etapa a ser seguida é a caracterização das amostras de solos e das misturas dessas com os aditivos químicos empregados, gerando parâmetros indispensáveis para os estudos subseqüentes de avaliação da resistência mecânica, como o teor ótimo de umidade e o peso específico seco máximo.

Nesse contexto, um novo material de construção surge com potencialidade de uso como estabilizante em pavimentos de estradas florestais, visto que é gerado no processo de recuperação da soda cáustica, durante o cozimento da madeira de eucalipto, na indústria de celulose. Trata-se de um resíduo sólido conhecido pelo nome de "grits".

Constituiu objetivo deste artigo caracterizar geotecnicamente amostras de solos e misturas solo"grits", com vistas à obtenção de parâmetros preliminares indispensáveis à execução de ensaios de resistência mecânica, durabilidade e resiliência, com vistas à aplicação das misturas em estradas florestais. Os objetivos específicos foram: (i) analisar a influência da adição de diversos teores do resíduo na plasticidade dos solos; e (ii) estudar a influência da adição de teores do resíduo nos parâmetros ótimos de compactação dos solos.

\section{MATERIAL E MÉTODOS}

\subsection{Solos}

Os solos do presente estudo são provenientes da Zona da Mata Norte de Minas Gerais, sendo as amostras oriundas de taludes da área de influência da cidade de Viçosa. A primeira amostra, de textura argilosa, foi coletada nas proximidades da estação de tratamento de água de Viçosa, e aqui designada por solo 1; geotecnicamente trata-se de um solo residual maduro de gnáisse e, pedologicamente, de um Latossolo
Vermelho-Amarelo. A segunda amostra, de textura arenosa, foi coletada na Vila Secundino, no Campus da Universidade Federal de Viçosa (UFV) e aqui designada por solo 2; geotecnicamente, trata-se de um solo residual jovem de gnáisse com horizonte $\mathrm{C}$ profundo, de coloração acinzentada.

\subsection{Resíduo industrial}

O resíduo industrial "grits" foi cedido pela empresa Celulose Nipo-Brasileira (CENIBRA S/A), situada na região de Ipatinga, Minas Gerais, Brasil. Trata-se de um resíduo sólido, de coloração acinzentada e granulometria diversificada. De acordo com a caracterização química efetuada por Machado et al. (2003), o "grits" apresenta, em sua composição química, cerca de $20 \%$ de cálcio, dos quais $42 \%$ se encontram na forma de óxido de cálcio $(\mathrm{CaO})$, que é um componente significativo para a estabilização de solos.

\subsection{Coleta, secagem e estocagem das amostras de solo e de resíduo}

A primeira etapa deste trabalho foi direcionada à coleta das amostras de solo, empregando-se picareta e pá. As amostras foram colocadas em sacos plásticos de $1.000 \mathrm{~L}$, perfazendo-se o total de $300 \mathrm{~kg}$ para cada solo. Após o transporte das amostras para o Laboratório de Engenharia Civil (LEC) da UFV, procedeu-se à secagem ao ar livre e à sombra. Em seguida, realizou-se o processamento das amostras na peneira de abertura nominal de 4,8 mm e estocagem em tambores metálicos, para posterior utilização nos ensaios geotécnicos.

O resíduo "grits" foi coletado no aterro de resíduos da empresa CENIBRA S/A em um quantitativo aproximado de $500 \mathrm{~kg}$, transportado para o Laboratório de Engenharia Civil da UFV e estocado em tambores, sem prévia secagem à sombra, objetivando não comprometer suas propriedades hidráulicas. O teor de umidade em que o "grits" se encontrava era de aproximadamente $18 \%$.

\subsection{Teores de "grits" empregados}

Com base em estudo prévio de estabilização desenvolvido com solos da CENIBRA S/A, definiramse os seguintes traços de "grits" em relação à massa de solo seco: $0,4,8,12,16,20,24$ e $28 \%$.

\subsection{Misturas solo-resíduo}

As misturas solo-resíduo foram realizadas adicionando-se, primeiramente, o resíduo "grits" aos 
solos, seguido por homogeneização manual e peneiramento (\# 4,8 mm) dessa mistura. Posteriormente, adicionou-se água em quantitativos definidos previamente, para atingir teores de umidade desejáveis, procedendo-se a uma nova homogeneização, peneiramento e acondicionamento das misturas em sacos plásticos hermeticamente fechados, para posterior uso.

\subsection{Ensaios e classificações de solos realizados}

Realizaram-se os seguintes ensaios, normatizados peloDepartamento de Infra-estrutura de Transportes (DNIT), com as amostras de solo e misturas solo-"grits": (i) análise granulométrica (DNIT, 1994a-ME 051); (ii) peso específico dos grãos do solo (DNIT, 1994b ME 093); (iii) limite de liquidez (DNIT, 1994c-ME 122); (iv) limite de plasticidade (DNIT, 1994d - ME 082); e (v) compactação nas energias referentes aos ensaios Proctor intermediário e Proctor modificado (DNIT, 1994e - ME 129). As classificações das amostras de solo pelos sistemas Transportation Research Board (TRB) e Unifield Soil Classification (USC), bem como pela metodologia Miniatura, Compactado e Tropical (MCT), preconizada por Nogami e Villibor (1995), também foram realizadas.

\section{RESULTADOS E DISCUSSÃO}

\subsection{Caracterização e classificação das amostras de solo}

No Quadro 1, encontram-se os resultados de caracterização dos solos 1 e 2. Destaque seja feito à elevada fração argila do solo 1 (60\%), caracterizando-o como representativo de um solo argilo-areno-siltoso, e à predominância da fração areia (74 \%) no solo 2, qualificandoo como material areno-silto-argiloso. Devido à sua textura, obteve-se na amostra 1 um valor de índice de plasticidade relativamente elevado (IP $=33 \%$ ), com base nos valores do DNIT-CLA 259 (1996), antevendo-se a presença de um solo mais suscetível ao aumento de volume do que aquele representado pela amostra $2(\mathrm{IP}=8 \%)$.

As classificações dos solos 1 e 2, segundo os sistemas TRB e USC, bem como pela metodologia MCT, encontram-se descritas no Quadro 2. Nogueira (1961) descreveu os solos do grupo A4 a A7 do sistema TRB, caso do solo 1 , como os que apresentam comportamento como material de subleito, de regular a mau, e os solos grosseiros, caso do solo 2, A-2-4, de excelente a bom. Murrieta (1994) descreveu que solos do grupo $\mathrm{MH}$, caso do solo 1 em questão, apresentam compactação regular a ruim, alta compressibilidade, e não são adequados como material de base de pavimentos rodoviários; por sua vez, solos do grupo SC (solo 2) apresentam compactação boa a regular, pequena à média compressibilidade e qualidade como material de base regular a ruim.

Com relação à classificação MCT dos solos, Nogami e Villibor (1995) descreveram que o solo 1 (LG') se trata de material com comportamento laterítico argiloso e o solo 2 (NA'), de material com comportamento nãolaterítico arenoso.

As características de compactação do solo 1 e do solo 2 são ilustradas na Figura 1. Nessa figura é possível observar que a amostra 1 possui curvas que se assemelham a uma parábola perfeita, com pontos de máximos bem definidos, verificando-se, também, que os parâmetros ótimos de compactação, teor ótimo de umidade $\left(\mathrm{W}_{\text {oft }}\right)$ e peso específico seco máximo $\left(\gamma_{\mathrm{dmáx}}\right)$ indicam um conteúdo maior de água no solo 1, em razão da presença superior de finos que governam a forma e a amplitude de sua curva de compactação. O solo 2, por possuir maior porcentual de areia, é mais suscetível à compactação; explicação para isso está, segundo Raghavan e Alvo et al. (1990), no fato de que suas partículas se arranjem mais acirradamente do que nos solos com predominância da fração argila. Menores valores de $\mathrm{W}_{\text {ot }}$ e maiores valores de $\gamma_{\text {dmáx }}$, à medida que o teor de areia aumenta, foram relatados, também, por Pacheco e Dias Júnior (1990) e Figueiredo (1998).

Quadro 1 - Caracterização geotécnica das amostras de solo Table 1 - Geotechnical characterization of soil samples

\begin{tabular}{lcc}
\hline Parâmetro & Solo 1 & Solo 2 \\
\hline Granulometria $(\%)-$ Segundo Escala & ABNT (1986) \\
Fração pedregulho $(\phi>2 \mathrm{~mm})$ & 0 & 0 \\
Fração areia $(0,06 \mathrm{~mm}<\phi \leq 2 \mathrm{~mm}$ & 28 & 74 \\
Fração silte $(0,002 \mathrm{~mm}<\phi \leq 0,06 \mathrm{~mm})$ & 12 & 11 \\
Fração argila $(\phi \leq 0,002 \mathrm{~mm})$ & 60 & 15 \\
\hline Peso Específico dos Sólidos $\left(\gamma_{\mathrm{s}}\right)-\mathrm{kN} / \mathrm{m}^{3} 27,63$ & 27,01 \\
\hline Limites de Atterberg $(\%)$ & & \\
Limite de liquidez $(\mathrm{LL})$ & 73 & 27 \\
Limite de plasticidade $(\mathrm{LP})$ & 40 & 19 \\
Índice de plasticidade $(\mathrm{IP})$ & 33 & 8 \\
\hline
\end{tabular}

Quadro 2 - Classificações TRB, USC e MCT das amostras de solos

Table 2-TRB, USC and MCT classification of soil samples

\begin{tabular}{lccc}
\hline \multirow{2}{*}{ Amostra } & \multicolumn{3}{c}{ Classificação } \\
\cline { 2 - 4 } & TRB & USC & MCT \\
\hline Solo 1 & A-7-5 (20) & MH & LG' \\
Solo 2 & A-2-4 (0) & SC & NA' \\
\hline
\end{tabular}

R. Árvore, Viçosa-MG, v.30, n.4, p.629-635, 2006 

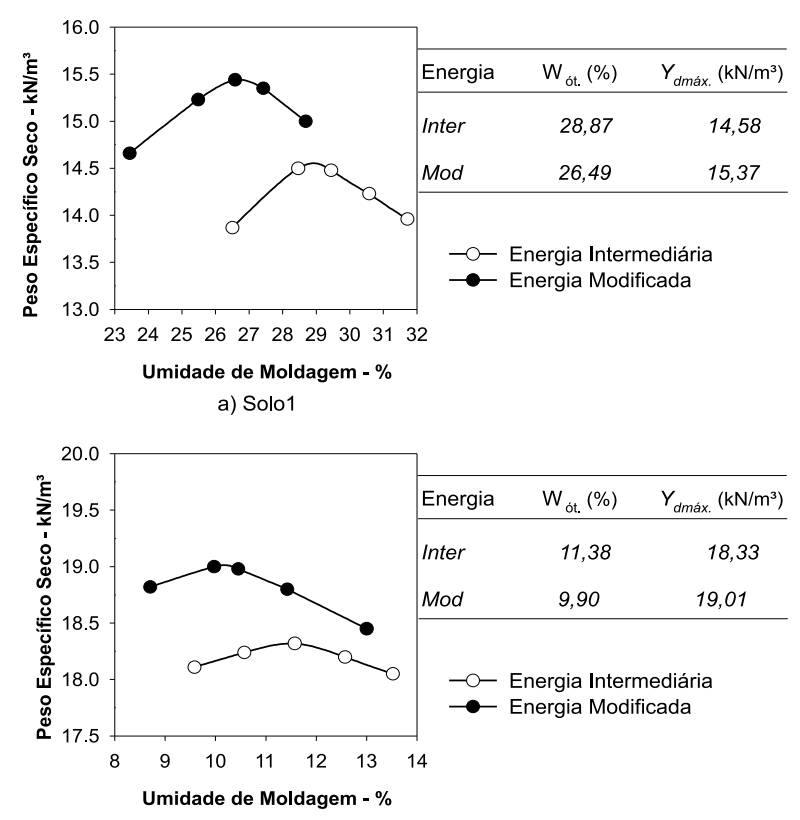

b) Solo2

Figura 1 - Resultados dos ensaios de compactação dos solos. Figure 1 - Soil compaction test data.

\subsection{Caracterização das misturas solo-"grits"}

A granulometria do resíduo "grits”, que se aproxima da granulometria do solo 2 , é constituída por $76 \%$ de partículas correspondentes à fração areia, dos quais $41 \%$ se enquadram como areia grossa e $35 \%$ como areia fina. As frações silte e argila correspondem a $18 \% \mathrm{e}$ a fração pedregulho, a $6 \%$. O "grits" apresentou-se como material não plástico (NP), com baixo peso específico aparente $\operatorname{seco}\left(\gamma_{\mathrm{s}}=16,38 \mathrm{kN} / \mathrm{m}^{3}\right)$.

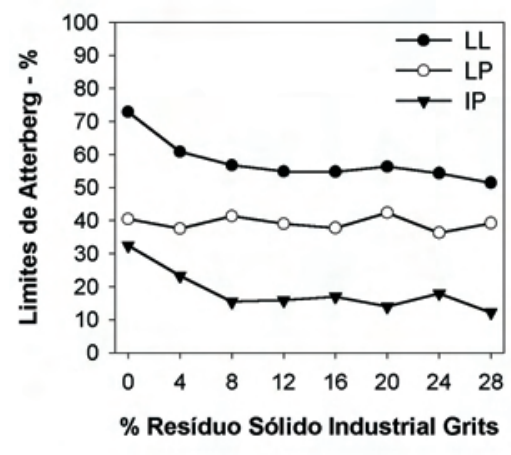

a) Misturas Solo1-Grits
O Quadro 3 contém os resultados dos ensaios dirigidos à análise da influência dos teores de "grits" sobre o peso específico dos sólidos $\left(\gamma_{\mathrm{s}}\right)$ das amostras de solo. Notou-se, tanto no solo 1 quanto no solo 2 , que a adição de "grits" reduziu o $\gamma \mathrm{s}$, conforme esperado, haja vista que o resíduo apresenta baixo valor para esse índice físico. De acordo com relatos de Pinto (2000), o $\gamma_{\mathrm{s}}$ varia pouco de solo para solo e, por si só, não possibilita identificá-los, mas é necessário para o cálculo de outros índices, exemplo das famílias de curvas de saturação de solos e de suas misturas com aditivos.

Na Figura 2, encontram-se os valores dos limites de consistência dos solos 1 e 2 e de suas misturas com diferentes teores de "grits". Nas misturas solo 1-“grits", a adição de resíduo reduziu o valor de LL do solo 1 de $73 \%$ para a faixa compreendida entre 51 a $61 \%$, sendo esse último limite referente ao teor de $28 \%$ de "grits".

Quadro 3 - Valores de $\gamma_{\mathrm{s}}$ das misturas solo-"grits" Table $3-\gamma_{s}$ values for soil-grits mixtures

\begin{tabular}{ccc}
\hline \% Grits & \multicolumn{2}{c}{ Peso específico dos sólidos $\left(\gamma_{\mathrm{s}}\right)-\mathrm{kN} / \mathrm{m}^{3}$} \\
\cline { 2 - 3 } & Solo1-“Grits” & Solo2-“Grits” \\
\hline 0 & 27,63 & 27,01 \\
4 & 26,99 & 25,72 \\
8 & 26,93 & 25,55 \\
12 & 27,37 & 25,50 \\
16 & 27,69 & 26,88 \\
20 & 27,69 & 26,80 \\
24 & 26,99 & 26,70 \\
28 & 26,87 & 25,79 \\
\hline
\end{tabular}

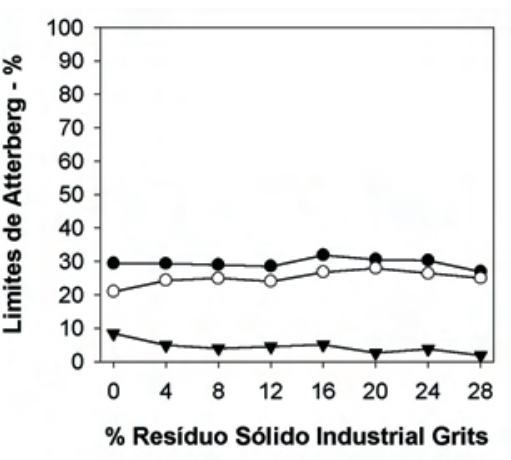

b) Misturas Solo2-Grits

Figura 2 - Limites de Atterberg dos solos e das misturas solo-"grits".

Figure 2 - Atterberg limits of soils and soil-grits mixtures.

R. Árvore, Viçosa-MG, v.30, n.4, p.629-635, 2006 
O valor de IP do solo 1 teve ligeira tendência à queda, quando da adição de teores de "grits", ocorrendo redução de $33 \%$, valor do solo 1 em sua forma natural, para a faixa de 12 a $23 \%$. O efeito do resíduo nos parâmetros de plasticidade do solo 2 foi menos significativo, com ligeira tendência ao aumento do LL e do LP, gerando como conseqüências reduções dos valores de IP, que foram mais acentuados no teor de $28 \%$ de "grits" (redução observada de $63 \%$ no IP).

A curva de compactação de solos consiste na representação gráfica do peso específico seco em função do teor de umidade e é função do tipo de solo, do método de compactação e da energia empregada (RICO e DEL CASTILLO, 1974; SOUZA, 1980). O efeito proporcionado pelo emprego de aditivos químicos nos solos pode ser o mais variado possível, indo desde o deslocamento das curvas de compactação até a mudanças na forma destas.
Em se tratando das misturas solo-"grits", notase na Figura 3 que a adição do resíduo ao solo 1 proporcionou deslocamentos das curvas para a esquerda e para acima, ou seja, menores valores de umidade de moldagem e maiores valores de peso específico seco foram obtidos, quanto maior o porcentual de "grits" nas misturas, em ambas as energias de compactação empregadas. Pode-se associar esse comportamento à ocorrência de reações de troca catiônica entre o resíduo "grits" e a fração fina do solo, o que proporcionou agregação de partículas argilosas e redução das suas características de plasticidade. Portanto, diminuíram as exigências de umidade para se atingir determinado arranjo de partículas, do que resultaram densidades secas mais elevadas. Outro fato, também associado ao comportamento das curvas das misturas solo 1"grits", foi a adição de fração mais grosseira, do resíduo, na granulometria do solo 1 , que com a redução da fração argila levou a pesos específicos secos maiores.
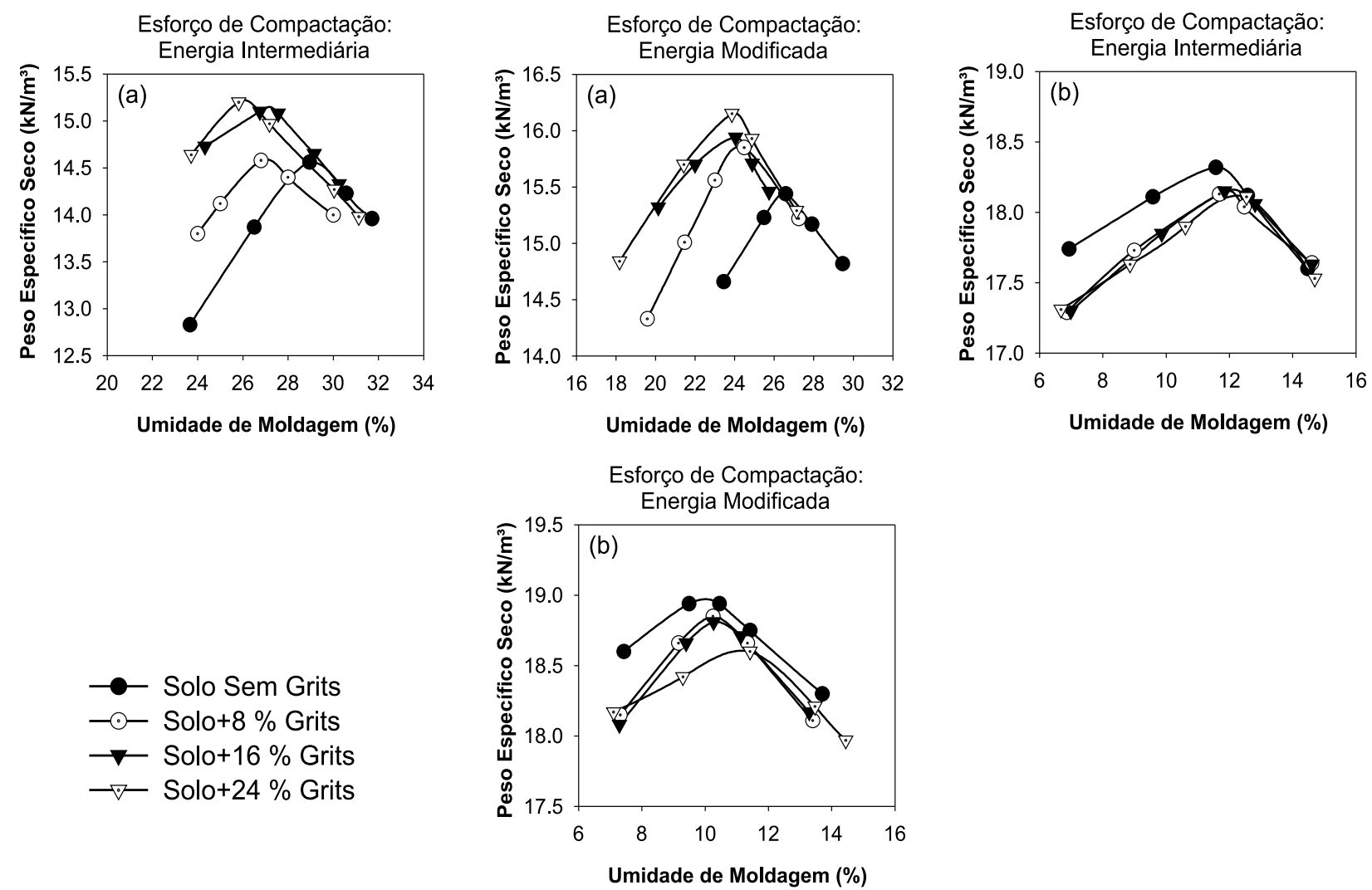

Figura 3 - Influência do "grits" nas curvas de compactação dos solos 1 (a) e 2 (b). Figure 3 - Influence of grits on compaction curves for soils 1 (a) and 2 (b). 
Nas misturas solo 2-"grits" houve comportamentos contrários aos observados nas misturas solo 1-"grits", ou seja, deslocamento da curva do solo natural para a direita (maior umidade de moldagem) e para baixo (menor peso específico aparente seco). Esse comportamento pode ser explicado pelo fato de que, ao se adicionar "grits" à amostra 2 , de textura predominantemente arenosa, estar-seá incluindo na nela, um aditivo de peso específico dos sólidos inferior e textura muito semelhante à sua.

Nos pontos de máximos das curvas de compactação, definem-se os parâmetros ótimos de compactação, ou seja, o teor ótimo de umidade $\left(\mathrm{W}_{\text {ot }}\right)$ e o peso específico aparente seco máximo $\left(\gamma_{\text {dmáx }}\right)$. No Quadro 4 , apresentamse os valores desses parâmetros nas misturas solo 1"grits", em que a adição de "grits", em todos os tratamentos, levou a quedas nos teores ótimos de umidade e aumentos nos pesos específicos aparentes secos, tomando-se como base o solo sem adição de resíduo e se referindo a ambas as energias de compactação empregadas. De forma análoga, no Quadro 5 são apresentados os resultados obtidos nas misturas solo 2"grits", em que ocorreu comportamento diferenciado, ou seja, aumento nos teores ótimos de umidade e quedas, na maioria dos casos, nos pesos específicos aparentes secos máximos.

\section{CONCLUSÕES}

Os resultados obtidos neste trabalho permitiram concluir que a adição de grits aos solos pesquisados nas dosagens estudadas alterou significativamente as características de plasticidade e os parâmetros de compactação das amostras de solos analisadas, observando-se maiores efeitos nas misturas solo1-“grits".

Quadro 4 - Parâmetros ótimos de compactação das misturas solo 1-"grits"

Table 4-Optimum compaction parameters of soil 1-grits mixtures

\begin{tabular}{|c|c|c|c|c|}
\hline \multirow[t]{3}{*}{ Misturas } & \multicolumn{4}{|c|}{ Energia de compactação } \\
\hline & \multicolumn{2}{|c|}{ Intermediária } & \multicolumn{2}{|c|}{ Modificada } \\
\hline & $\gamma_{\mathrm{dmax}}\left(\mathrm{kN} / \mathrm{m}^{3}\right)$ & $\mathrm{W}_{\text {ot. }}(\%)$ & $\gamma_{\text {dmáx }}\left(\mathrm{kN} / \mathrm{m}^{3}\right)$ & $\mathrm{W}_{\text {ot. }}(\%)$ \\
\hline Solo 1 sem "grits" & 14,58 & 28,87 & 15,37 & 26,49 \\
\hline Solo $1+4 \%$ "grits" & 15,04 & 27,85 & 15,57 & 25,72 \\
\hline Solo $1+8 \%$ "grits" & 15,01 & 27,55 & 15,85 & 24,47 \\
\hline Solo $1+12 \%$ "grits" & 14,89 & 27,37 & 15,97 & 24,28 \\
\hline Solo $1+16 \%$ "grits" & 15,14 & 27,06 & 16,03 & 23,39 \\
\hline Solo $1+20 \%$ "grits" & 15,08 & 26,29 & 16,14 & 23,09 \\
\hline Solo $1+24 \%$ "grits" & 15,20 & 25,85 & 16,16 & 22,75 \\
\hline Solo $1+28 \%$ "grits" & 15,45 & 24,45 & 16,19 & 22,57 \\
\hline
\end{tabular}

Quadro 5 - Parâmetros ótimos de compactação das misturas solo 2-"grits"

Table 5 - Optimum compaction parameters of soil 2-grits mixtures

\begin{tabular}{lcccc}
\hline Misturas & \multicolumn{3}{c}{ Energia de compactação } \\
\cline { 2 - 5 } & $\gamma_{\mathrm{dmax}}\left(\mathrm{kN} / \mathrm{m}^{3}\right)$ & Intermediária & $\gamma_{\mathrm{dmax}}\left(\mathrm{kN} / \mathrm{m}^{3}\right)$ & Modificada \\
\cline { 2 - 5 } & 18,33 & $\mathrm{~W}_{\text {ot. }}(\%)$ & 19,01 & $\mathrm{~W}_{\text {ot. }}(\%)$ \\
\hline Solo 2 sem "grits" & 18,21 & 11,38 & 9,90 \\
Solo 2 + 4 \% "grits" & 18,14 & 11,47 & 19,02 & 9,98 \\
Solo 2 + 8 \% "grits" & 18,17 & 11,57 & 18,86 & 10,24 \\
Solo 2 + 12 \% "grits" & 18,15 & 11,58 & 18,84 & 10,22 \\
Solo 2 + 16 \% "grits" & 18,16 & 11,92 & 18,81 & 10,33 \\
Solo 2 + 20 \% "grits" & 18,13 & 11,96 & 18,74 & 10,41 \\
Solo 2 + 24 \% "grits" & 18,04 & 12,37 & 18,59 & 11,29 \\
Solo 2 + 28 \% "grits" & & 12,41 & 18,65 & 11,26 \\
\hline
\end{tabular}

R. Árvore, Viçosa-MG, v.30, n.4, p.629-635, 2006 


\section{AGRADECIMENTOS}

Ao CNPq e à FAPEMIG, pelo apoio financeiro, bem como à CAPES e à CENIBRA S/A, respectivamente, pela concessão da bolsa de doutorado ao primeiro autor e pelo fornecimento do resíduo "grits".

\section{REFERÊNCIAS BIBLIOGRÁFICAS}

ASSOCIAÇÃO BRASILEIRA DE NORMAS TÉCNICAS - ABNT. NBR 7181: solo: análise granulométrica. Rio de Janeiro: 1984. 13 p.

DEPARTAMENTO NACIONAL DE INFRAESTRUTURA DE TRANSPORTES - DNIT. ME 051: solos: análise granulométrica. Rio de Janeiro: 1994a. $12 \mathrm{p}$.

\section{DEPARTAMENTO NACIONAL DE INFRA-} ESTRUTURA DE TRANSPORTES - DNIT. ME 093: solos: determinação da densidade real. Rio de Janeiro: 1994b. 4 p.

DEPARTAMENTO NACIONAL DE INFRAESTRUTURA DE TRANSPORTES - DNIT. ME 122: solos: determinação do limite de liquidez: método de referência e método expedito. Rio de Janeiro: 1994c. 7 p.

\section{DEPARTAMENTO NACIONAL DE INFRA-} ESTRUTURA DE TRANSPORTES - DNIT. ME 082: solos: determinação do limite de plasticidade. Rio de Janeiro: 1994d. 3 p.

\section{DEPARTAMENTO NACIONAL DE INFRA-} ESTRUTURA DE TRANSPORTES - DNIT. ME 129: solos: compactação utilizando amostras não-trabalhadas. Rio de Janeiro: 1994e. 7 p.

DEPARTAMENTO NACIONAL DE INFRAESTRUTURA DE TRANSPORTES - DNIT. CLA 259: classificação de solos tropicais para finalidades viárias utilizando corpos-de-prova compactados em equipamentos miniatura. São Paulo: 1996.6 p.

FERNANDES, D.C.M. Viabilidade do uso de alcatrão de madeira de eucalipto na estabilização de solos residuais para fins rodoviários. 2000. 124 f. Tese (Doutorado em Ciências Florestais)-Universidade Federal de Viçosa, Viçosa, 2000.
FIGUEIREDO, L.H.A. Propriedades físicas e mecânicas de um Latossolo Roxo submetido a diferentes sistemas de manejo. 1998. 68 p. Dissertação (Mestrado em Solos e Nutrição de Plantas)-Universidade Federal de Lavras, Lavras, 1998.

MACHADO, C.C.; PEREIRA, R.S.; PIRES, J.M.M. Influência do tratamento térmico do resíduo sólido industrial grits na resistência mecânica de um latossolo para pavimentos de estradas florestais. Revista Árvore, v. 27, n. 4, p. 543-550, 2003.

MURrieta, P. Apostila de mecânica dos solos: 1aㅡ parte. Brasília: Universidade de Brasília, 1994. Não paginado.

NOGAMI, J.S.; CORREIA, F.C.; VILLIBOR, D.F. Perspectivas da aplicação do solo-cal em rodovias: exemplos de pistas experimentais. In: REUNIÃO ABERTA DA INDÚSTRIA DA CAL, 4., 1980, São Paulo. Anais... São Paulo: ABPC, 1980. p. 212-234. (Boletim, 12).

NOGAMI, J.S.; VILLIBOR, D.F. Pavimentação de baixo custo com solos lateríticos. São Paulo: Villibor, 1995. 240 p.

NOGUEIRA, C. Pavimentação. Rio de Janeiro: Ao Livro Técnico, 1961. 485 p.

PACHECO, A.A.R.; DIAS JÚNIOR, M.S. Estudo comparativo de métodos de campo e laboratório aplicados à confecção de blocos em adobe. Ciência e Prática, v. 14, n. 2, p. 176-190, 1990.

PINTO, C.S. Curso básico de mecânica dos solos em 16 aulas. São Paulo: Oficina de Textos, 2000. 247 p.

RAGHAVAN, G.S.V.; ALVO, P.; MCHYES, E. Soil compaction in agriculture: a review toward managing the problem. Advances in Soil Science, v. 11, p. 1-36, 1990.

RICO, A.; DEL CASTILLO, H. La ingenieria de suelos en las vias terrestres. México: Limusa, 1974. v.1. 455 p.

SOUZA, M.L. Pavimentação rodoviária. 2 . ed. Rio de Janeiro: DNER: Instituto de Pesquisa Rodoviária, 1980. 364 p. (Livros Técnicos e Científicos).

R. Árvore, Viçosa-MG, v.30, n.4, p.629-635, 2006 